\title{
A MICRODROPLET-BASED CAPACITIVE SENSING MATRIX FOR TACTILE APPLICATIONS
}

\author{
B. Nie ${ }^{1}$, R. $\mathrm{Li}^{1}$, J.D. Brandt ${ }^{2}$, and T. Pan ${ }^{1 *}$ \\ ${ }^{1}$ Department of Biomedical Engineering, University of California, Davis, USA \\ ${ }^{2}$ Department of Ophthalmology, University of California, Davis, Health System, USA
}

\begin{abstract}
In this paper, we present a highly sensitive microdroplet-based interfacial capacitive sensor matrix device, achieving ultrahigh sensitivity of $0.4 \mathrm{nF} / \mathrm{kPa}$ and minimal detectable pressure of $33 \mathrm{~Pa}$ at a $3 \times 3 \times 0.2 \mathrm{~mm}^{3}$ packaging. The microdroplet-based sensor is comprised of an array of nanoliter ionic droplets sandwiched between two flexible substrates with patterned transparent electrodes. The sensing principle primarily relies on high elasticity of the sensing droplet and large capacitance presented at the electrode-electrolyte interface. Theoretical analyses and experimental investigations on several design parameters are thoroughly conducted to characterize. Finally, the microdroplet-based pressure sensor is successfully utilized for tactile sensing applications, such as Braille characters scanning and non-invasive cardiovascular pressure recording.
\end{abstract}

\section{INTRODUCTION}

Artificial tactile sensing devices have been extensively researched for their potential utilities in medicines and robotics, in which a wide spectrum of sensing mechanisms have been introduced and investigated, including resistive, piezoelectric and capacitive sensing [1-4]. Among those, elastomeric resistive materials have been frequently employed in building such arrays for their low cost and easy manufacturability [1]. Alternatively, piezoelectric nanomaterial has been incorporated into a pressure sensitive matrix with a sub-millimeter resolution for tactile imaging [2]. Capacitive sensing leads another trend in the development of artificial skins. Conventional capacitive tactile array, using a parallel plate configuration, has been devised for both pressure and force mapping [3]. In more recent effort, a flexible capacitive pressure sensor has been implemented with a microstructured elastomer layer as the sensing element for rapid mechanical responses (in the range of milliseconds) [4].

Latest development on iontronic materials (e.g., ionic liquids) renews long interest in utilizing ionic solutions to regulate the electronic transport at the electrolyte-electrode interface. Upon the solid-liquid contact, an electric double layer (EDL) immediately establishes with an ultrahigh unit-area capacitance on the order of $10 \mu \mathrm{F} / \mathrm{cm}^{2}$ at the atomic interface, which is more than thousand times greater than that of the solid-state counterpart (up to $2 \mathrm{nF} / \mathrm{cm}^{2}$ ) $[5,6]$. It has been shown that the iontronic device can be utilized in transistor or sensors, where the EDL exhibits ultrahigh-density charge accumulation while avoiding reactions by its electrochemical inertness within the potential windows [7-9].

This report present a novel microdroplet-based iontronic sensor array (MISA), utilizing the ultrahigh EDL capacitance at the solid-liquid interface, for tactile sensing applications. Figure 1 illustrates the transparent MISA device of $12 \times 12$ elements with the spatial resolution of $1 \mathrm{~mm}$. Benefiting from the ultrahigh unit-area capacitance of the EDL, we can achieve a device sensitivity of $0.4 \mathrm{nF} / \mathrm{kPa}$ and minimal detectable pressure of $33 \mathrm{~Pa}$ at a $3 \times 3 \times 0.2 \mathrm{~mm}^{3}$ packaging, which to our best knowledge is the most sensitive capacitive pressure sensor at its dimension. As the demonstrations of utilities of the iontronic devices, the MISA has successfully resolved the surface topology and detected the dynamic wrist pulses throughout cardiovascular cycles.

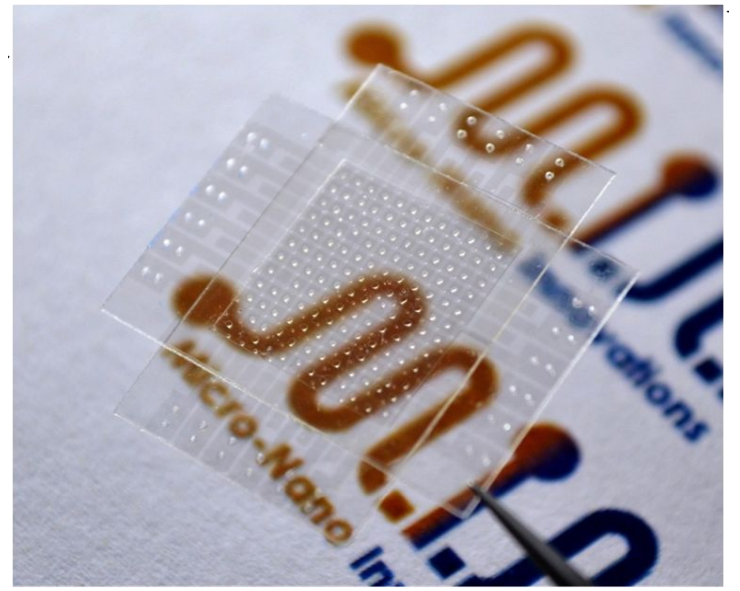

Fig. 1: Photography of the sensor array of $12 \times 12$ elements with the spatial resolution of $1 \mathrm{~mm}$.

\section{THEORY}

The MISA device consists of two flexible films with micropatterned transparent electrodes and one spacing layer hosting an array of nanoliter ionic droplets. Figure 2 exhibits the interfacial capacitive sensing principle. The direct ionic droplet-electrode contact immediately establishes the EDL, which possesses a remarkable interfacial capacitance (on the order of $10 \mu \mathrm{F} / \mathrm{cm}^{2}$ ). Under external loads, the flexible surfaces experience mechanical deformation, resulting in circumferential expansion of the droplet-electrode contact. The corresponding capacitive change over the increased contact area can be detected electronically. Device sensitivity can be modeled both mechanically and electrically. The measured EDL capacitance can be directly related to the area of the droplet-electrode contact, as the invariant unit-area capacitance can be experimentally determined. On the other hand, the mechanical deformation of the membrane can be well defined in the classic mechanic theory [10]. It is worth noting that the interfacial capacitive sensing principle offers an ultrahigh capacitive sensitivity, which is more than thousand times greater than that of the solid-state counterpart, contributed mainly from the nanoscopic charge separation in EDL. The relationship between the measurable capacitive change ( $\Delta \mathrm{C}$ ) and the contact pressure applied $(\Delta \mathrm{P})$ can be derived from the capacitive sensing principle:

$$
\Delta C \approx C_{0} \times\left[\frac{\Delta P}{\alpha \times H \times T^{3} / L^{4}}+\left(\frac{\Delta P}{\alpha \times H \times T^{3} / L^{4}}\right)^{2}\right]
$$

where $C_{0}$ and $H$ indicate the initial capacitance and height of the sensing cell, respectively, and the constant $\alpha=5 \times E \times\left(1-v^{2}\right)^{-1}$ represents the mechanical deformation of the sensing membrane, influenced by the Young's modulus $E$ and Poisson ratio $v$. As can be seen, the relationship between the capacitive change $(\Delta C)$ and the contact pressure $(\Delta \mathrm{P})$ is highly dependent on the spatial resolution $L$ and the membrane thickness $T$. In addition, the gravitational effect has been neglected in our consideration, as the microdroplet dimensions are considerably less than that of the capillary length (of $\sim 1.8 \mathrm{~mm}$ ). 


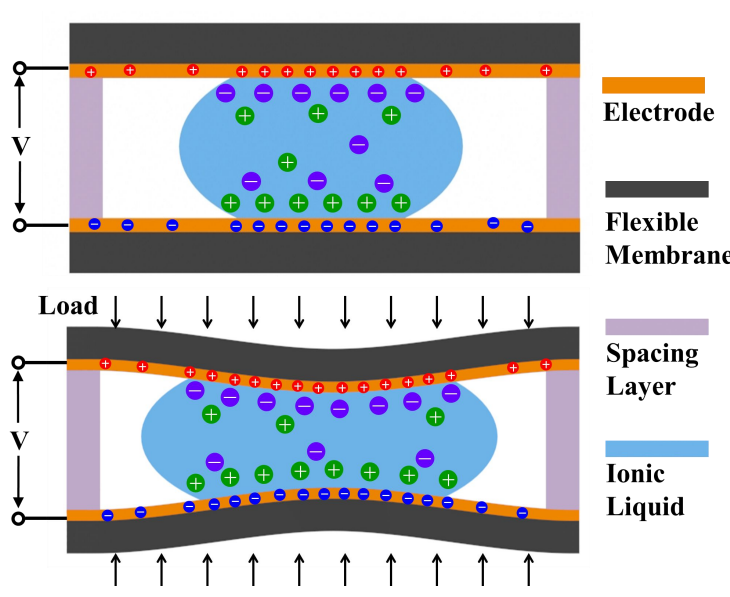

Fig. 2 The interfacial capacitive sensing principle. The EDL capacitance is established at the ionic droplet-electrode contact (top). The contact area expands circumferentially, which is caused by the membrane deformation under external loads and results in an increase in the EDL capacitance (bottom).

\section{METHODS}

\section{Device fabrication}

The fabrication process starts with micropatterning of conductive ITO electrodes on the flexible PET films using standard photolithography followed by wet etching. In the subsequent step, dry-film photoresist is laminated and photopatterned into a micropillar array on the electrode surface, serving as the supporting structure. To accurately position the microdroplets, surface wettability patterning is applied. A hydrophobic oligomer layer of PDMS is transfer-printed onto both electrode surfaces. Subsequently, using a microfluidic impact printing technique, nanoliter droplets of ionic liquid will be sequentially deposited into a grid matched to the hydrophilic spots formed by the wettability patterning. Prior to the final assembly, two electrode films are aligned face-to-face with the conductive patterns positioned orthogonally to each other, forming a grid of capacitances at the crossover points where the ionic droplet array sits in. The top and bottom layers are then bonded together through the plasma activated hydroxyl groups of the PDMS oligomer layers.

\section{Electrical and mechanical characterization}

Experimental investigations of the device sensitivity have been conducted on individual sensing units of the iontronic microdroplet array. The measurement stage is comprised of a force gauge with 1 $\mathrm{mN}$ resolution (DFS, Chatillon) amounted on a computer-controlled step motor (VT-80, PImicos) with a spatial resolution of $400 \mathrm{~nm}$, from which mechanical loads and displacements can be controlled and monitored simultaneously. The corresponding capacitive changes are directly recorded through an LCR meter (4284A, Agilent). In the characterization of the responsive time, an electromagentically driven pin actuator (Panasonic KX-P1150), powered by a pulsed voltage signal, has been used to apply the periodic contact pressure to the sensor surface. The output signals are measured by a custom circuitry.

\section{RESULTS}

\section{Sensing Fluid}

As a vital part of the interfacial sensing device, physical properties of the sensing droplet have to satisfy following criteria: high ionic concentration (ensuring high electrical conductance and interfacial capacitance), polarized molecular structure (reversible elasticity on the hydrophobic surfaces) and low fluidic viscosity (allowing rapid mechanical response). Three types of ionic fluids have been considered, such as aqueous electrolytes (e.g., $\mathrm{NaCl}$ electrolyte solution), organic solvent solutions (e.g. $\mathrm{KClO}_{4} / \mathrm{PEO}$ ), and ionic liquids, which are commonly investigated in electrochemical processes [11]. Aqueous and solvent-based electrolytes are typically highly evaporative under room conditions and it becomes extremely challenging to maintain the constant electrical performance as both the volume and the physical properties change over time. As an emerging alternative, ionic liquids, consisting of an organic anion or cation, exhibit high electrical conductivity, low volatility, and tunable viscosity [12]. In the report, IL of 1-ethyl-3-methylimidazolium tricyanomethanide has been selected as the sensing fluid for the MISA.

\section{Mechanical-to-capacitive sensitivity}

As aforementioned, the overall mechanical-to-electrical sensitivity $(\Delta \mathrm{C} / \Delta \mathrm{P})$ can be determined by the geometrical confinements (the spatial resolution $L$ and height $H$ of each sensing unit), and the material properties of the membrane, e.g. the thickness $T$, Young's modulus $E$ and Poisson ratio $v$. Among those, the spatial resolution of the sensing unit play the most important role, followed by the thickness of the sensing membrane. Experimental investigations have been conducted to verify the above theoretical predications.
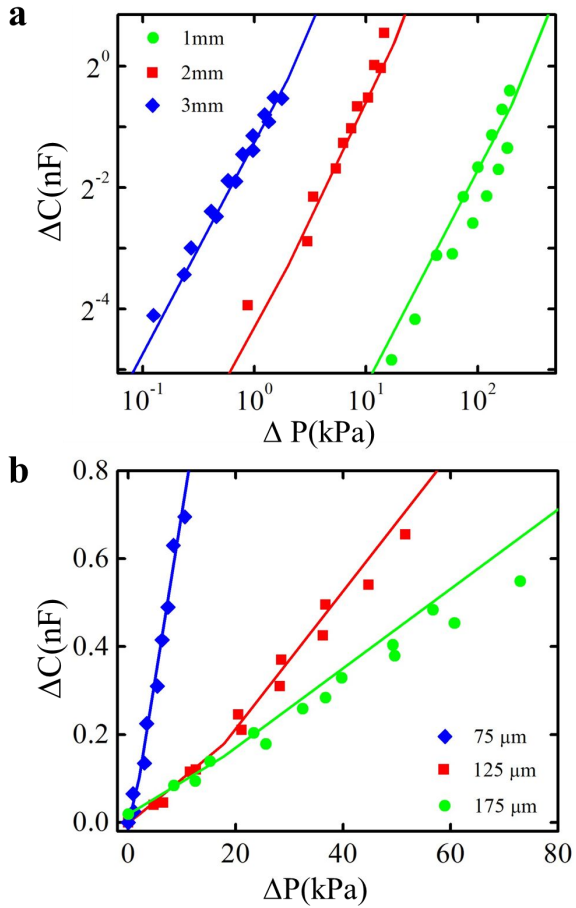

Fig. 3 Influences of spatial resolution $\boldsymbol{a}$ and membrane thickness $\boldsymbol{b}$ on the device sensitivity.

Figure 3 shows the experimental measurements on the device sensitivity with various geometrical designs of spatial resolutions and membrane thicknesses, in which the measurement results (dots) are plotted against the theoretical predications from Equation 1 (curves). As shown in Fig. 3a, the sensitivity exhibits a strong dependence (minor 4 th power) on the spatial resolution. By varying the spatial resolution from $1 \mathrm{~mm}$ to $3 \mathrm{~mm}$ with a constant membrane thickness of $75 \mu \mathrm{m}$, the sensitivity can be improved from $3.9 \mathrm{pF} / \mathrm{kPa}$ to $433.7 \mathrm{pF} / \mathrm{kPa}$ (more than 100 -fold increase), and the device achieves the highest sensitivity with the largest initial capacitance (of 2.3nF) among all the capacitive sensors at the same dimension. In addition, the minimal detectable pressure of $33 \mathrm{~Pa}$ is 
characterized on the sensor with the highest sensitivity.

Moreover, the membrane thickness plays another notable role in the device performance, as the sensitivity is inversely related to the 3rd power of the thickness. As plotted in Fig. 3b, by adjusting the membrane thickness from $75 \mu \mathrm{m}$ to $175 \mu \mathrm{m}$, with a fixed spatial resolution of $2 \mathrm{~mm}$, the thinner membrane (of $75 \mu \mathrm{m}$-thick) shows a higher sensitivity of $77.7 \mathrm{pF} / \mathrm{kPa}$, while the thicker devices (of $175 \mu \mathrm{m}$-thick) membranes exhibit a lower sensitivity of $7.8 \mathrm{pF} / \mathrm{kPa}$. Furthermore, the targeted dynamic range can be tuned by the geometrical constrains. For instance, in the most sensitive design ( $3 \mathrm{~mm}$ in resolution and $75 \mu \mathrm{m}$ in thickness), the maximal pressure is around $7 \mathrm{kPa}$, while the design of $2 \mathrm{~mm}$ in resolution and $175 \mu \mathrm{m}$ in thickness can extend the measurement range up to $450 \mathrm{kPa}$. Overall, the spatial resolution and the membrane thickness of the MISA could be the determinant factors in the sensor performance (i.e., device sensitivity and dynamic range), allowing highly customizable sensors for a wide range of specifications and applications.

\section{Response Time}

Experiments have been conducted to characterize the response time of the MISA devices. A pulsed contact pressure (of $\sim 1.4 \mathrm{kPa}$ ) in the frequency ranging from $10 \mathrm{~Hz}$ to $100 \mathrm{~Hz}$ has been applied to the device surface through an electromagnetically driven pin actuator. Both the driving voltages to the actuator and the capacitive changes are recorded. As shown in Fig. 4, the capacitive changes of the sensor repeats in the same frequency to the corresponding voltages applied to the pin actuator, suggesting that the sensor can response to the pressure in the frequency up to $100 \mathrm{~Hz}$. It is worth noting that the distortion of the recorded pressure signals likely attributes to the open-loop operation of the load applied by the actuator (i.e., the rapid rise edge and slow recovery phase of the capacitive readings).
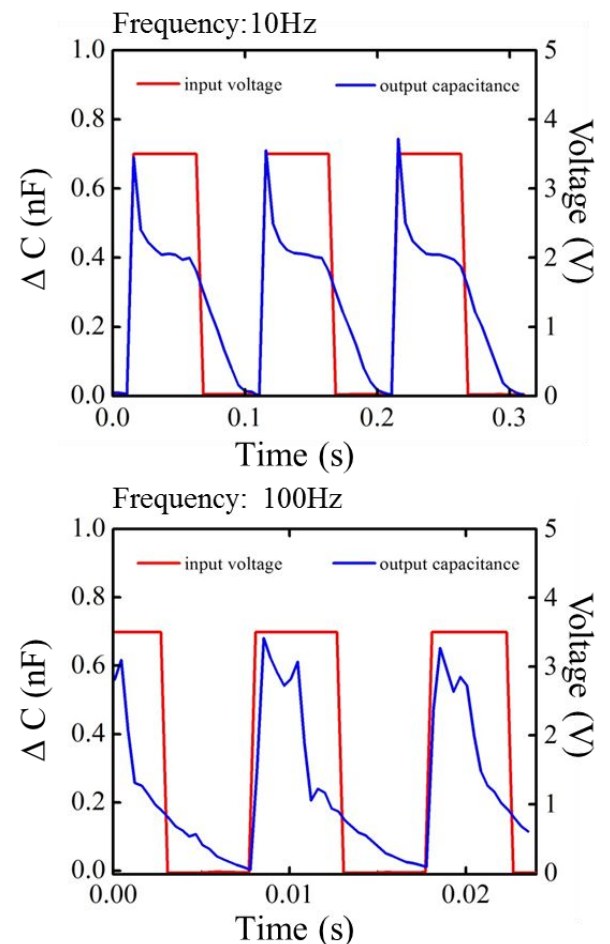

Fig. 4 The time-resolved sensor response measurements under repetitive mechanical loads in the frequency of $10 \mathrm{~Hz}$ and $100 \mathrm{~Hz}$ (red curves indicate the input voltage to drive actuator, and the blue curves are the output capacitance measured from a single sensing unit of the MISA device).

\section{Surface Topology Mapping}

To demonstrate the flexibility and adaptability of the MISA devices to artificial tactile applications, we have configured a sensor array to detect fine surface topology, such as Braille letters. As can be seen in Fig. 5, the custom MISA consists of $2 \times 3$ pixels with the spatial resolution of $2.3 \mathrm{~mm}$ (to match with the standard Braille letters), and can be worn in a fingertip set up. For the fingertip reading of Braille texts, a gentle contact pressure has been applied to the text surface by the finger. The raised dotted impressions of each Braille character cause the membrane deformation in the corresponding droplet sensing units, which can be subsequently detected by the change of the interfacial capacitance. As shown in bottom panel of Fig. 5, using the finger-amounted MISA device, the letter of "BRAILLE" has been successfully resolved, in which each pressure reading is converted to a digital colorimetric scale. Digital recording of the tactile sensing can be further processed and transmitted into audible readings, and thus, it can be of potential use for Braille education for visually impaired patients.

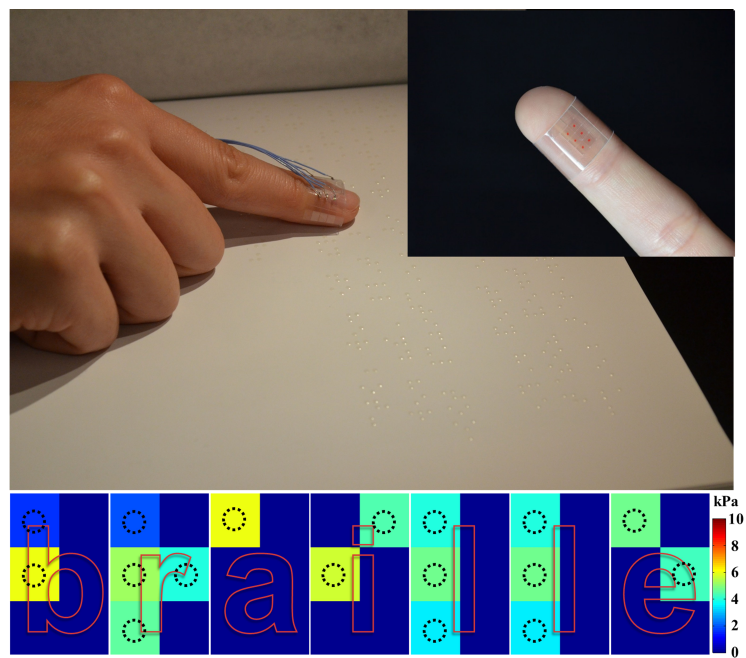

Fig. $52 \times 3$ MISA device, amounted onto a fingertip, is configured to resolve raised dots of the "BRAILLE" characters in a Braille textbook.

\section{Wrist Pulse Recording}

Furthermore, we have utilized the ultrahigh device sensitivity and rapid response time of the iontronic droplet sensors to non-invasive cardiovascular pressure recording. An MISA device of $5 \times 5$ array with the spatial resolution of $3 \mathrm{~mm}$ has been positioned in contact with the skin above the radial artery and fixed by a plastic wristband (Fig. 6a). Real-time pulse recording has been performed by scanning all the sensing elements covering the skin area of $15 \times 15 \mathrm{~mm}^{2}$ at the sampling frequency of $1 \mathrm{kHz}$ in each unit. The MISA device enables two important functions in the pulse recording. First of all, the sensor spatially maps the pulse on the skin surface, from which the sites of the maximal pressure variations can be located. Comparing the pressure mapping results (Fig. 6b) with the sensor position (Fig. 6a), the pressure sensing units right above the radial artery provide the highest capacitive recordings (marked as I, II, III) as expected, and thus, closely reflects the cardiovascular pressure readings using the tonometry principle. In the following step, the pressure wave forms are continuously tracked from these optimal sensing positions. Fig. 6c shows the continuous pulse recordings from the three pixels, respectively. As can be seen, the 
maximal pulse variation is around $1.2 \mathrm{kPa}$ recorded by Sensor II. Fig. $6 \mathrm{~d}$ provides a close-up analysis of a single cardiac cycle, which has been characterized into three peaks $\left(\mathrm{P}_{1}, \mathrm{P}_{2}\right.$ and $\left.\mathrm{P}_{3}\right)$. These maxima are caused by traveling waves of the systolic phase and diastolic phase of the blood pressure conducted in the elastic cardiovascular vessels [13]. Clinically significant parameters, such as the radial augmentation index, e.g. AI $\left(=\mathrm{P}_{2} / \mathrm{P}_{1}\right)$, can be directly extracted and computed from the maximal pulse recordings, which can be potentially used to screen the arterial compliance [14]. Moreover, the radial pulse waveforms recorded at the optimal sites can be further processed to estimate the central aortic pressure and cardiac output, which reflects the important cardiovascular events and the health states. Though a similar measurement has been conducted recently through a single-channel capacitive sensor, the IMA offers the combined advantage of simultaneous pressure mapping of the optimal recording area and continuous tracking of the blood pressure waveform, in addition to its flexible transparent packaging [4]. In this fashion, the MISA can serve as a flexible sensing device that is highly attractive for the emerging wearable health monitoring applications, in comparison with the conventional invasive cardiovascular monitoring.

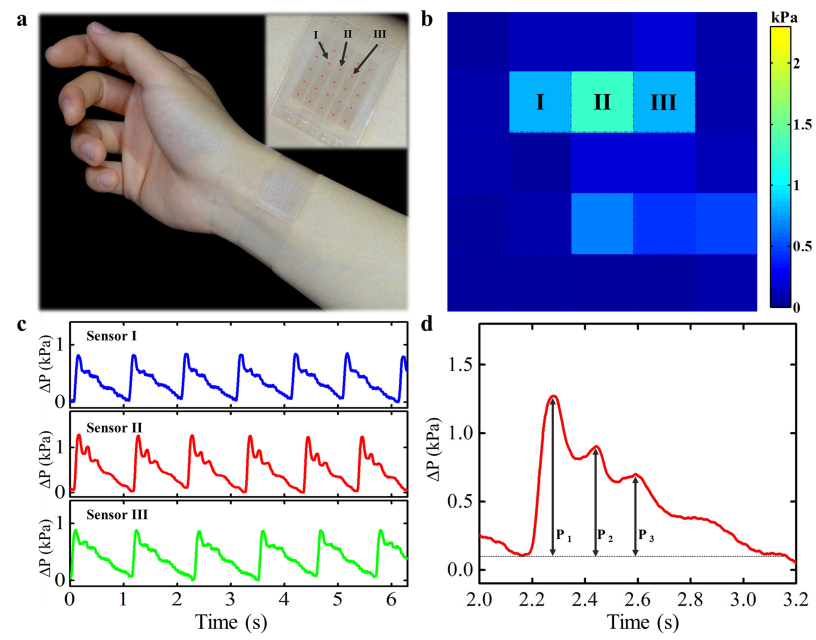

Fig. 6 Real-time wrist pulse measurements. a, illustration of a transparent MISA device of $5 \times 5$ array with each pixel size of $3 \times 3 \mathrm{~mm}^{2}$ embedded in a wrist band for pressure wave recording. $\boldsymbol{b}$, the spatial distributions of the pulse intensities mapped by the MISA. The highest pressure variation readings, marked as I, II and III, are located above the radial artery, in corresponding to the positions of the three units in $\boldsymbol{a}$. $\boldsymbol{c}$, time-resolved strongest pulses recorded by the marked sensing units in $\boldsymbol{a}$ and $\boldsymbol{b}$, respectively. $\boldsymbol{d}$, a close-up view of one pulse signal recorded by sensor II in $c$, in which $P_{1}, P_{2}$ and $P_{3}$ represent the three consecutive peaks of the pressure wave in each cardiovascular cycle.

\section{CONCLUSIONS}

In this paper, we have presented a novel microdroplet-based iontronic sensor array (MISA) with a very simple architecture and tunable sensitivities. The interfacial sensing principle utilizes the presence of large unit-area EDL capacitance at the elastic droplet-electrode contact. A theoretical model has been proposed to analyze the influences of the geometrical parameters (e.g. spatial resolution and membrane thickness) on the device sensitivity. Taking advantage of ultrahigh sensitivity, fast mechanical response, simple fabrication, mechanical flexibility and optical transparency, the iontronic microdroplet sensors are expected to be employed in a wide range of applications, including robotics, medical prosthetics, surgical instruments, video gaming and wearable computing

\section{ACKNOWLEDGEMENTS}

This work is in part supported by the NSF CAREER Award (ECCS-0846502) to TP. BN and RL acknowledge the fellowship support from China Scholarship Council (CSC). Authors would also like to acknowledge the travel support generously provided by the Transducer Research Foundation.

\section{REFERENCES}

[1] K. Takei, T. Takahashi, J. C. Ho, H. Ko, A. G. Gillies, P. W. Leu, R. S. Fearing and A. Javey," Nanowire active-matrix circuitry for low-voltage macroscale artificial skin”, Nature Materials, 9, 10 (2010).

[2] W. Wu, X. Wen and Z. L. Wang, "Taxel-Addressable Matrix of Vertical-Nanowire Piezotronic Transistors for Active and Adaptive Tactile Imaging", Science, 340 (2013).

[3] D. J. Lipomi, M. Vosgueritchian, B. C. Tee, S. L. Hellstrom, J. A. Lee, C. H. Fox and Z. Bao, , "Skin-like pressure and strain sensors based on transparent elastic films of carbon nanotubes", Nature Nanotechnology, 6 (2011).

[4] G. Schwartz, B. C.-K. Tee, J. Mei, L. Appleton, D. H. Kim, H. Wang and Z. Bao, "Flexible polymer transistors with high pressure sensitivity for application in electronic skin and health monitoring", Nature Communications, (2013).

[5] H. Yuan, H. Shimotani, A. Tsukazaki, A. Ohtomo, M. Kawasaki, and Y. Iwasa, "High-Density Carrier Accumulation in $\mathrm{ZnO}$ Field-Effect Transistors Gated by Electric Double Layers of Ionic Liquids", Advanced Functional Materials, 19, 7 (2009).

[6] M. J. Madou, "Fundamentals of Microfabrication: The Science of Miniaturization", Second Edition, (2002).

[7] J. T. Ye, S. Inoue, K. Kobayashi, Y. Kasahara, H. T. Yuan, H. Shimotani and Y. Iwasa, "Liquid-gated interface superconductivity on an atomically flat film", Nature Materials, 9 (2010).

[8] M. Armand, F. Endres, D. R. MacFarlane, H. Ohno and B. Scrosati, "Ionic-liquid materials for the electrochemical challenges of the future", Nature Materials, 8 (2009).

[9] B. Li, S. Dalgleish, Y. Miyoshi, H. Yoshikawa, M. M. Matsushita and K. Awaga, "Electric double layers allow for opaque electrodes in high performance organic optoelectronic devices", Apply Physics Letters, 101(2012).

[10] S. P. Timoshenko and S. Woinowsky-Krieger, "Theory of Plates and Shells", 1959.

[11] A. Lewandowski, A. Olejniczak, M. Galinski and I. Stepniak, "Performance of carbon-carbon supercapacitors based on organic, aqueous and ionic liquid electrolytes", Journal of Power Sources, 195 (2010).

[12] M. Galinski, A. Lewandowski and I. Stepniak, "Ionic liquids as electrolytes", Electrochimica Acta, 51(2006).

[13] I. B. Wilkinson, N. H. Mohammad, S. Tyrrell, I. R. Hall, D. J. Webb, V. E. Paul, T. Levy and J. R. Cockcroft, "Heart rate dependency of pulse pressure amplification and arterial stiffness", American Journal of Hypertension, 15(2002).

[14] S. Motohiro and K. Kazuomi, "Review: Role of the augmentation index in hypertension", Therapeutic Advances in Cardiovascular Disease, 2(2008).

\section{CONTACT}

*T.Pan, tel: +1-530-754-9508; tingrui@ucdavis.com 